\title{
Monolithic Add-Drop Multiplexers in Fused Silica Fabricated by Femtosecond Laser Direct Writing
}

\author{
Vítor A. Amorim, João M. Maia, D. Alexandre, and P. V. S. Marques
}

\begin{abstract}
The fabrication of optical add-drop multiplexers in fused silica is demonstrated, for the first time to our knowledge, using the femtosecond laser direct writing technique. To achieve this, a Mach-Zehnder interferometer configuration was used for the signal routing by the implementation of 3-dB directional couplers, along with Bragg grating waveguides for wavelength selectivity. The fabrication of all individual devices required was optimized. The behavior of the fabricated add-drop multiplexer was characterized at around $1550 \mathrm{~nm}$, where a 3-dB bandwidth of $0.19 \pm$ $0.01 \mathrm{~nm}$ was obtained along with an intrachannel and adjacent interchannel crosstalk of -30 and $-20 \mathrm{~dB}$ at $\Delta \lambda= \pm 0.75 \mathrm{~nm}$, respectively. This study shows that such complex devices can be manufactured by femtosecond laser direct writing, with future improvements being discussed.
\end{abstract}

Index Terms-Add-drop multiplexer, femtosecond laser, integrated optics, laser materials-processing applications, optical device fabrication, wavelength division multiplexing.

\section{INTRODUCTION}

W AVELENGTH-DIVISION multiplexing (WDM) for telecommunication purposes requires spectral filters for adding and dropping channels within a signal. These functions can be performed by Bragg gratings implemented in the arms of a Mach-Zehnder interferometer (MZI), resulting in an optical add-drop multiplexer (OADM) [1].

Integrated OADMs based on this configuration can be fabricated using photolithographic techniques together with UV photoinscription of Bragg gratings and phase trimming because these allow high quality devices with low insertion losses as well as low intra and inter-channel crosstalk. A more recent

Manuscript received February 6, 2017; revised April 12, 2017 and June 5, 2017; accepted June 9, 2017. Date of publication June 14, 2017; date of current version July 11, 2017. This work was supported in part by the Calouste Gulbenkian Foundation through the Stimulus to Scientific Research Program under Grant 141773, in part by the Project "NanoSTIMA: Macro-toNano Human Sensing: Towards Integrated Multimodal Health Monitoring and Analytics/NORTE-01-0145-FEDER-000016," which is financed by the North Portugal Regional Operational Programme (NORTE 2020) under the PORTUGAL 2020 Partnership Agreement, and in part by the European Regional Development Fund. (Corresponding author: Vitor A. Amorim.)

V. A. Amorim, J. M. Maia, and P. V. S. Marques are with the Centre for Applied Photonics, Instituto de Engenharia de Sistemas e ComputadoresTecnologia e Ciência, Porto 4169-007, Portugal, and also with the Department of Physics and Astronomy, University of Porto, Porto 4169-007, Portugal (e-mail: vitor.a.amorim@inesctec.pt; joao.m.maia@inesctec.pt; psmarque@fc.up.pt).

D. Alexandre is with the Centre for Applied Photonics, Instituto de Engenharia de Sistemas e Computadores-Tecnologia e Ciência, Porto 4169-007, Portugal, and also with the Department of Physics, University of Trás-os-Montes e Alto Douro, Vila Real 5001-801, Portugal (e-amil: daniel@utad.pt).

Color versions of one or more of the figures in this paper are available online at http://ieeexplore.ieee.org.

Digital Object Identifier 10.1109/JLT.2017.2715118 fabrication alternative is to use a femtosecond laser to write the optical path directly on the material [2]. While photolithographic techniques offer significant advantages in scalability, cost, and circuit compactness, femtosecond laser direct writing can offer prototyping flexibility, without the need for masks or multiple fabrication steps, out of the plane geometries, and real-time optimizations that are not present in these traditional fabrication methods. This technique can be used in several materials [2] and already enabled the fabrication of many integrated optical devices, such as buried waveguides [3], Bragg grating waveguides (BGWs) [4], directional couplers [5], Y-junctions [6], and integrated lasers [7] among others. Femtosecond laser direct writing can also aid in the development of new solutions for the ongoing exponential growth trend in data communications. The implementation of multicore fibers (as opposed to single core), to scale the information transmitted by the number of cores, requires a complete toolbox of solutions and devices matching the characteristics of those fibers. To solve this problem a true 3D technique should be implemented, as opposed to photolithographic techniques that are inherently planar. Three-dimensional fan-out devices for multicore fiber coupling applications have already been reported with this technique [8]. Photolithographic techniques, with their maturity, should, however, not be discarded but complemented with femtosecond laser direct writing, since heterogeneous $2 \mathrm{D}$ and $3 \mathrm{D}$ photonic integration can bring diverse and complementary functions. An example of this is the orbital angular momentum multiplexers demonstrated using a hybrid 3D photonic integrated circuit that combines a silica photonic lightwave circuit with a 3D fan-out device [9]. Current 3D components have, however, limitations in density due to the large bending radius, associated with the relatively low index contrast, and the minimum distance allowed between waveguides, since coupling among adjacent waveguides is an issue. Future improvements in this area are expected. Due to the technique's inherent high spatial resolution and 3D capabilities the monolithic integration of optofluidic devices for sensing applications is also possible [10], [11].

Fig. 1 shows a schematic of a possible OADM configuration, which is based on a MZI and that has been investigated in this work. When this device is implemented between two telecommunication terminals a transmitted (input) signal, composed by several wavelength channels with a defined channel spacing, is split equally into two waveguides by the first directional coupler. Both signals are then guided to two identical BGWs that reflect the resonant wavelength to the drop port, where the channel can be collected and the information read. The remaining signal is 


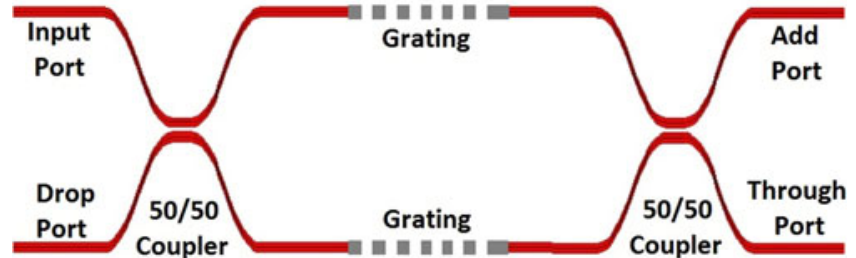

Fig. 1. Schematic of the optical add-drop multiplexer configuration.

transmitted and emerges at the through port, allowing the propagation of the remaining information channels. Similarly to the channel drop, new information at the resonant wavelength can be introduced to the output signal via the add port. The spectral behavior of the resonant channel is thus established mainly by the BGWs performance, while the broad spectral behavior of the device is defined by the achromaticity of the employed $3 \mathrm{~dB}$ splitters.

In this configuration the routing of the signals is guaranteed by the MZI; however, in order to perform this routing correctly both arms must have an identical optical path length. Since the phase difference between two signals that are reflected by the BGWs is given by:

$$
\Delta \emptyset=\frac{4 \pi n_{e f f} \Delta L}{\lambda},
$$

where $n_{\text {eff }}$ is the waveguide effective index, $\Delta L$ is the BGW positioning error given by the relative shift between both gratings on the arms of the MZI, and $\lambda$ the signal wavelength, it can be shown that for both signals to be roughly in phase $\left( \pm 10^{\circ}\right)$ the positioning error must be around/or smaller than 15 nm (for fused silica @ 1550 nm). Such an identical optical path length can be achieved repeatedly by post-fabrication real time trimming.

In this paper we report a systematic study of the fabrication and optimization of each component required to build an OADM, as well as the OADM itself. Even though the results have room for improvement, this demonstrates that complex devices can be fully fabricated by the femtosecond laser direct writing technique. The characteristics of BGWs are investigated as a function of the writing beam duty cycle and its fabrication parameters set for the implementation in OADMs. Directional couplers, that are required in the fabrication of the MZI, were also optimized for $3 \mathrm{~dB}$ splitting. For this, several geometries with different separation distances between interacting waveguides and different interaction lengths were tested. Finally, the optical components were implemented together to demonstrate, for the first time to our knowledge, a fully functional OADM at $1550 \mathrm{~nm}$ done by femtosecond laser direct writing.

\section{EXPERIMENTAL PROCEDURE}

To fabricate the integrated optical components an Amplitude Systèmes Satsuma HP fiber amplified femtosecond laser system, emitting a second harmonic beam at $515 \mathrm{~nm}$ with an approximate pulse duration of $250 \mathrm{fs}$ at $500 \mathrm{kHz}$, was used to induce the refractive index modification in homogeneous pure silica substrates (Suprasil). The laser beam was focused below

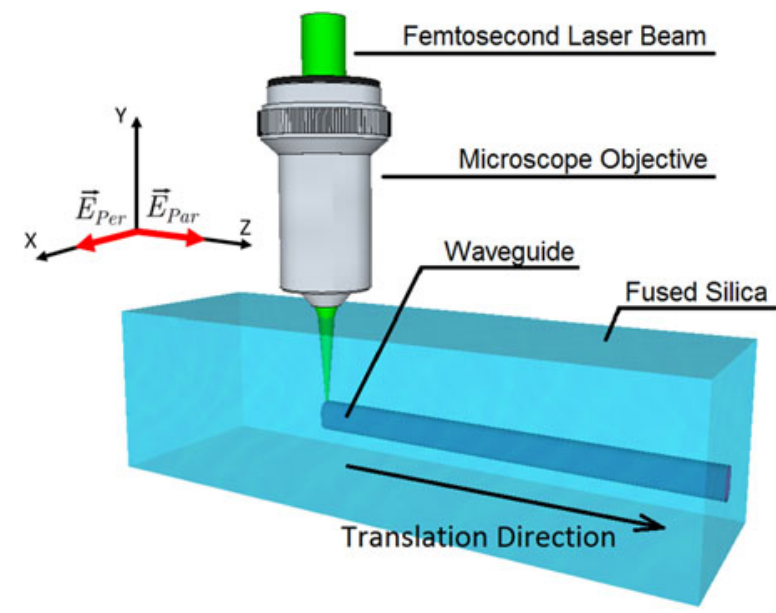

Fig. 2. Schematic of device fabrication using the transverse writing geometry, where $\vec{E}_{\mathrm{Par}}$ and $\vec{E}_{\mathrm{Per}}$ represent the parallel and perpendicular polarizations of the writing laser, respectively.

the substrate surface using a 0.55 numerical aperture aspherical lens (Newport 5722-A-H) mounted in a vertical piezo stage (PI P-725.4CD PIFOC). The substrate was mounted on X-Z Aerotech air-bearing linear stages (ABL10100-LN) and translated in relation to the beam focus, with a scanning direction orthogonal to the incident laser beam, as seen in Fig. 2 .

To characterize the fabricated components a tunable laser (Santec TSL-210V) was coupled to the device via a single-mode fiber (SMF-28) or V-grooves (from Precision Micro-Optics). The substrate was mounted on an Elliot Martock MDE881 with piezo control (Dali E-2100) and special holders for single and V-groove mounted fibers. V-grooves were glued to the final OADM with Norland optical adhesive 61 (NOA 61) using an UV light source (Hamamatsu LC8), while for the other test devices both input and output single-mode fibers were butt-coupled to the device using index matching (Cargille series: $\mathrm{AA}_{\mathrm{D}}^{25^{\circ} \mathrm{C}}=1.458 \pm 0.0002$ ), to minimize Fresnel reflections. To analyze the polarization dependent behavior, an inline fiber polarizer (Thorlabs ILP1550PM-APC) together with polarization maintaining fibers were used. The transmitted light was analyzed by a power meter (Exfo Optical System IQ-203 with an IQ-1600 module). Mode profiles were obtained with a $20 \times$ microscope objective and a CCD camera (Chameleon CMLN-13S2M-CS).

\section{EXPERIMENTAL RESULTS AND DISCUSSION}

Low loss optical waveguides are essential for the good performance of any guided wave optical circuit. In this work several parameters were optimized, namely the laser beam pulse energy, substrate translation velocity, writing depth, and writing beam polarization orientation in relation to the written waveguide axis (parallel and perpendicular, as demonstrated in Fig. 2). Fig. 3 shows insertion loss measured for waveguides fabricated with a linearly polarized beam whose polarization was parallel to the scanning direction, while varying the remaining parameters. It was found that the lowest insertion losses, at $1550 \mathrm{~nm}$, are originated at $250 \mathrm{~nJ}$ per pulse, with a scanning velocity of 

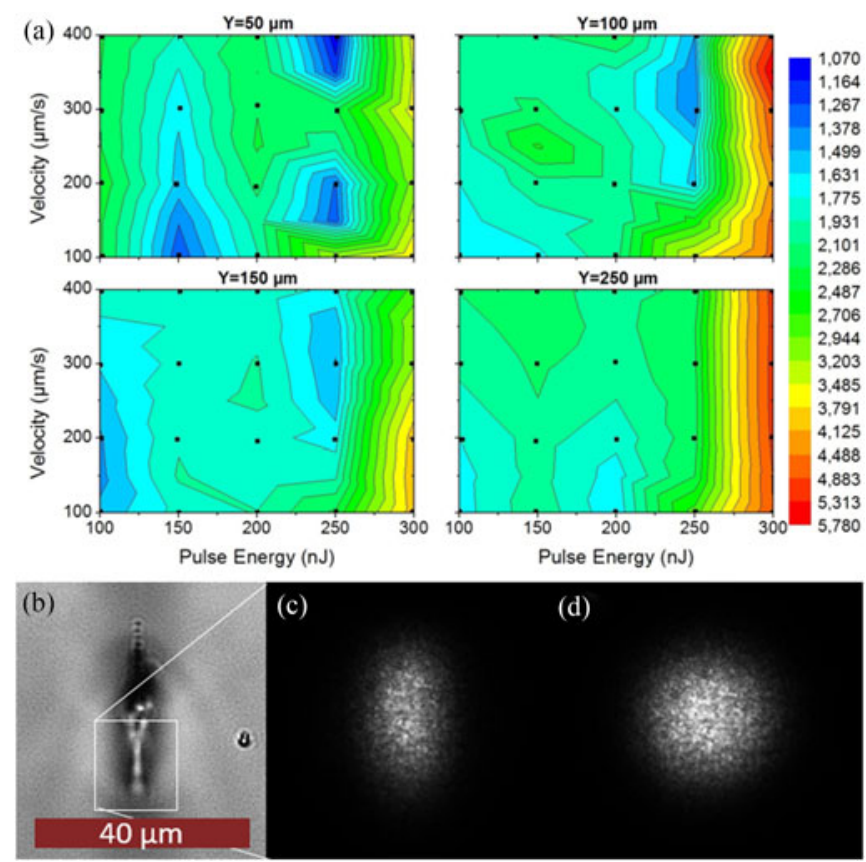

(c)

(d)

Fig. 3. (a) Insertion loss in $\mathrm{dB}$, at $1550 \mathrm{~nm}$, of the fabricated waveguides as a function of pulse energy and scan velocity for different depths and a parallel writing beam polarization. (b) Cross sectional microscope image and (c) mode profile, at $1550 \mathrm{~nm}$, of the waveguide written at a depth of $50 \mu \mathrm{m}$, with $250 \mathrm{~nJ}$ per pulse and a translation velocity of $400 \mu \mathrm{m} / \mathrm{s}$. (d) Mode profile of a SMF-28 fiber at $1550 \mathrm{~nm}$.

$400 \mu \mathrm{m} / \mathrm{s}$, and at a depth of $50 \mu \mathrm{m}$. These exposure conditions resulted in a total insertion loss and polarization dependent loss of $1.09 \pm 0.03 \mathrm{~dB}$ and $0.30 \pm 0.04 \mathrm{~dB}$ respectively, for $2.5 \mathrm{~cm}$ long waveguides in fused silica. At this wavelength the waveguides are single mode, and the propagated intensity mode field diameter (MFD) is $7.1 \times 12.3 \pm 0.2 \mu \mathrm{m}$ (along $\mathrm{x}$ and $\mathrm{y}$, respectively), resulting in calculated coupling losses (assuming a Gaussian distribution [12]) of $0.37 \pm 0.05 \mathrm{~dB}$ per facet with a SMF-28 fiber (MFD of $10.4 \mu \mathrm{m}$ ) and a propagation loss of $0.14 \pm 0.04 \mathrm{~dB} / \mathrm{cm}$, which are in line with the best results in literature [3], [13].

Bend loss was also investigated by measuring the added insertion loss of a S-bend in relation to a reference straight waveguide. The input and output waveguide offset, $\mathrm{S}$, as shown in the inset of Fig. 4, was tested between 125 and $500 \mu \mathrm{m}$. The S-bends investigated were arc shaped and the bend loss was studied by varying the arc radii between $3 \mathrm{~mm}$ and $80 \mathrm{~mm}$. The measured bend loss, which consists of transition loss at inflection points and loss due to the curvature itself [5], is plotted in Fig. 4 as a function of bend radius for input light at a wavelength of $1550 \mathrm{~nm}$ and an offset of $125 \mu \mathrm{m}$, since the loss per unit length does not depend on waveguide separation. As expected, the bend loss decreases dramatically with the increase of the bend radius, being negligible for bend radii larger than $50 \mathrm{~mm}$. For safety, the bend radius used in the fabricated devices was $80 \mathrm{~mm}$. The smoothness of the experimental data behavior should also be pointed out, showing that the fabrication process is reproducible as well as the quality of the measurements and fabricated waveguides.

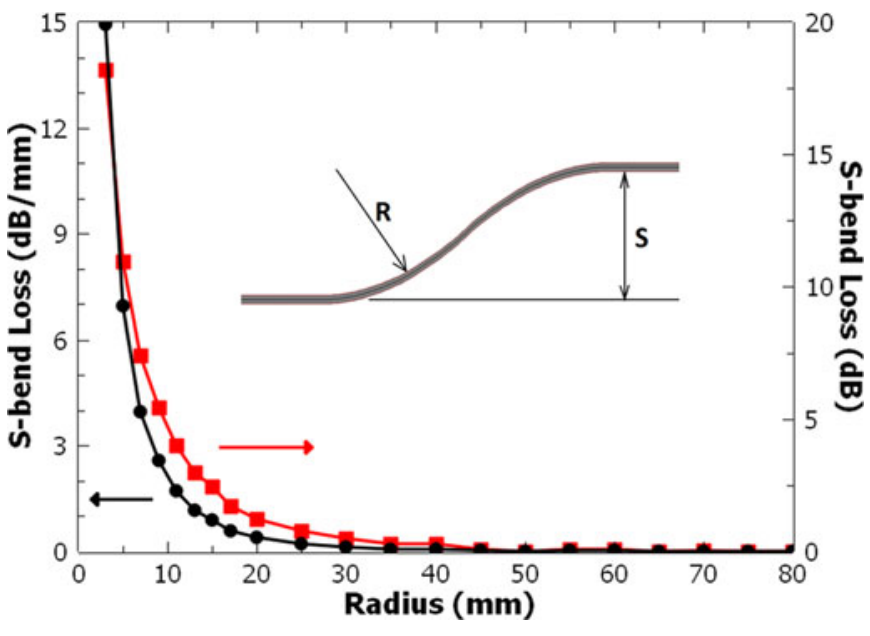

Fig. 4. Bend loss as a function of bend radius for $125 \mu \mathrm{m}$ input/output waveguide separation. Inset shows a schematic of a S-bend with two bends of radius $\mathrm{R}$ and a waveguide offset $\mathrm{S}$.

The bend loss results presented in this paper are in accordance with other works reported in the literature using this technique [14], [15]. Regarding Fukuda's work, it can be seen, however, that for higher pulse energies a much lower bend loss is achievable with a relatively low propagation loss of $\sim 0.25 \mathrm{~dB} / \mathrm{cm}$ (after annealing the waveguides). The reduction of bend loss was also shown by Eaton et al. by using a higher numerical aperture of 1.25 , due to a higher refractive index modification. Waveguides fabricated with this numerical aperture showed, however, an increased propagation loss of $0.6 \mathrm{~dB} / \mathrm{cm}$ and a smaller MFD of $7.1 \times 7.4 \mu \mathrm{m}$. Although the reduction of bend loss for smaller bend radii enables the fabrication of more compact devices, the waveguides that enable shorter S-bends have higher propagation losses, meaning that, depending on the device, the insertion losses may increase. Also, the combination of waveguides with low propagation loss and S-bends with low bend loss is possible, but with a large mode mismatch between sections, causing transition losses.

\section{A. Bragg Grating Waveguides}

A Bragg grating waveguide is a structure with a periodic perturbation of the refractive index that guides light while reflecting in a narrow range of wavelengths around the Bragg wavelength, given by:

$$
\lambda_{B}=2 n_{e f f} \Lambda
$$

where $\Lambda$ is the grating period.

The optimization of the BGWs was accomplished by using the same writing parameters as the optimized optical waveguides. In order to fabricate segmented waveguides the laser internal acousto-optic modulator (AOM) was controlled by a function generator (Stanford Research Systems DS345) as a fast on/off switch to enable the modulation of the femtosecond laser beam. Since the grating periodicity is given by:

$$
\Lambda=\frac{v}{f},
$$




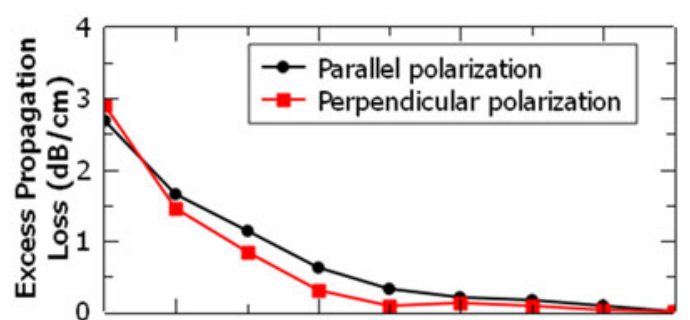

(a)

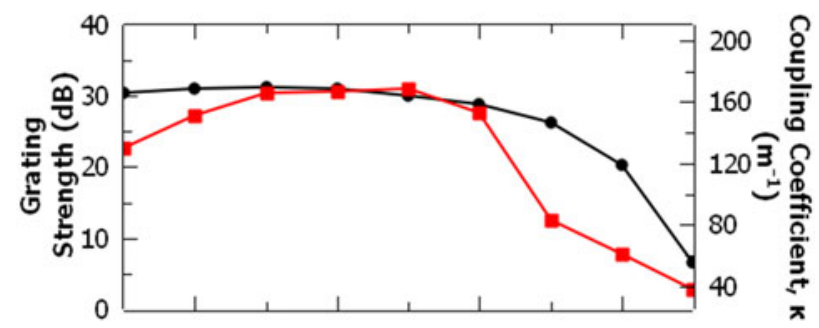

(b)

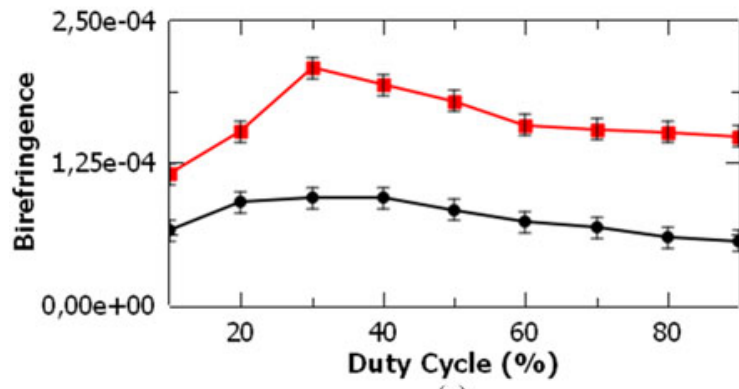

(c)

Fig. 5. (a) Excess propagation loss, (b) grating strength/coupling coefficient, and (c) birefringence as a function of duty cycle for BGWs fabricated with a beam polarization parallel and perpendicular to the writing direction.

where $v$ is the fixed scanning velocity and $f$ the function frequency, the Bragg wavelength was controlled by tuning the modulation frequency. In this study the function was set to a square waveform and the duty cycle varied. The BGWs were fabricated in $25 \mathrm{~mm}$ long substrates.

The aim of this study was to investigate the properties of the fabricated structures at around $1550 \mathrm{~nm}$, which were obtained with a grating periodicity of approximately $536.1 \mathrm{~nm}$ (first order grating). This value was obtained by fabricating several BGWs while varying the grating periodicity; by doing a linear fit to the results, and using an interpolation, it was possible to obtain the grating periodicity matching the desired wavelength.

In order to find the best fabrication parameters a study was made varying the square wave duty cycle (from 10 to $90 \%$ ) and writing beam polarization orientation (parallel and perpendicular to the scanning direction). As shown in Fig. 5, the AOM modulation duty cycle and writing beam polarization strongly affects the BGW properties. Fig. 5(a) shows the BGW excess propagation loss (propagation loss difference between a reference waveguide and a BGW at $1555 \mathrm{~nm}$ ) increasing monotonically for both writing polarizations, from 0 to $\sim 3 \mathrm{~dB} / \mathrm{cm}$, as the duty cycle decreases from 90 to $10 \%$. This is likely due to the increasing refractive index modulation [4]. As expected, this increase in refractive index modulation quickly improves the grating strength, with a maximum of $\sim 31 \mathrm{~dB}\left(\mathrm{~K} \approx 171 \mathrm{~m}^{-1}\right)$ being obtained around a duty cycle of $40 \%$, while higher duty cycles generated weaker gratings, reaching $\sim 5 \mathrm{~dB}$ for a duty cycle of $90 \%$, as seen in Fig. 5(b). Birefringence was also measured, as shown in Fig. 5(c), and found to be around 3 times larger with a perpendicular writing beam polarization when in comparison with parallel polarization. A maximum was obtained for a duty cycle around $30 \%$. This birefringence can arise from laser induced stresses and the formation of nanogratings aligned along preferential directions [16], and can be tuned (decreased even) using stress tracks parallel to the BGWs [17].

The coupling coefficient of these gratings is in accordance with the strongest gratings achieved using femtosecond laser direct writing in fused silica [18]. In this case, the gratings fabricated by Peter Zeil et al. achieved a maximum coupling coefficient of $280 \mathrm{~m}^{-1}$, a result $68 \%$ higher than those in this paper. Despite the fabrication parameters being very similar in both cases, the higher coupling coefficient can be attributed to a lower writing velocity $(10 \mathrm{~mm} / \mathrm{min} \approx 167 \mu \mathrm{m} / \mathrm{s}$, as opposed to the $400 \mu \mathrm{m} / \mathrm{s}$ used in this paper), since the laser fluence is higher, meaning a higher refractive index modification. The fabrication of gratings with a coupling coefficient of $1457 \mathrm{~m}^{-1}$, using this technique, has already been reported by Martin Ams et al. [19] in silicate glass, enabling much shorter device lengths with the same characteristics.

As Fig. 5 shows, a compromise must be made when choosing the AOM duty cycle for the optimization of the grating strength against the excess propagation loss and birefringence. From this study it can be seen that a $60 \%$ duty cycle offers a good combination of high grating strength $\left(\sim 29 \mathrm{~dB}, \mathrm{k} \approx 161 \mathrm{~m}^{-1}\right)$ and low excess propagation loss $(\sim 0.3 \mathrm{~dB} / \mathrm{cm})$, with a transition loss of $0.05 \pm 0.02 \mathrm{~dB}$ for the optimized waveguides. However, for the fabrication of the OADMs, a parallel polarization and a duty cycle of $40 \%$ were chosen due to the lower birefringence and grating size constraints $(20 \mathrm{~mm})$ created by the length of the complete device which decreases the grating strength.

\section{B. Directional Couplers}

The $3 \mathrm{~dB}$ directional couplers were fabricated with the geometry seen in Fig. 6(a), using the optimal parameters already discussed for the fabrication of optical waveguides and S-bends. In this particular study, the coupling region waveguide centerto-center separation distance was tested between 5 and $15 \mu \mathrm{m}$ and the interaction length between 0 and $12 \mathrm{~mm}$, resulting in 12 to $25 \mathrm{~mm}$ long devices. As shown in Fig. 6(b)-(d), the coupling ratio behavior for ports 3 and 4 as a function of interaction length is sinusoidal and its amplitude close to unity, as expected from identical waveguides. The coupling length was analyzed, where a monotonical increase is found for an increasing separation distance, as shown in Fig. 6(e). For a $5 \mu \mathrm{m}$ separation distance a coupling length around $0.5 \mathrm{~mm}$ is obtained, while for a $15 \mu \mathrm{m}$ separation distance the coupling length is approximately $5.6 \mathrm{~mm}$.

The insertion loss was also investigated for all directional couplers fabricated. Fig. 7 shows insertion loss as a function of interaction length for all separation distances tested. For a 
(a)
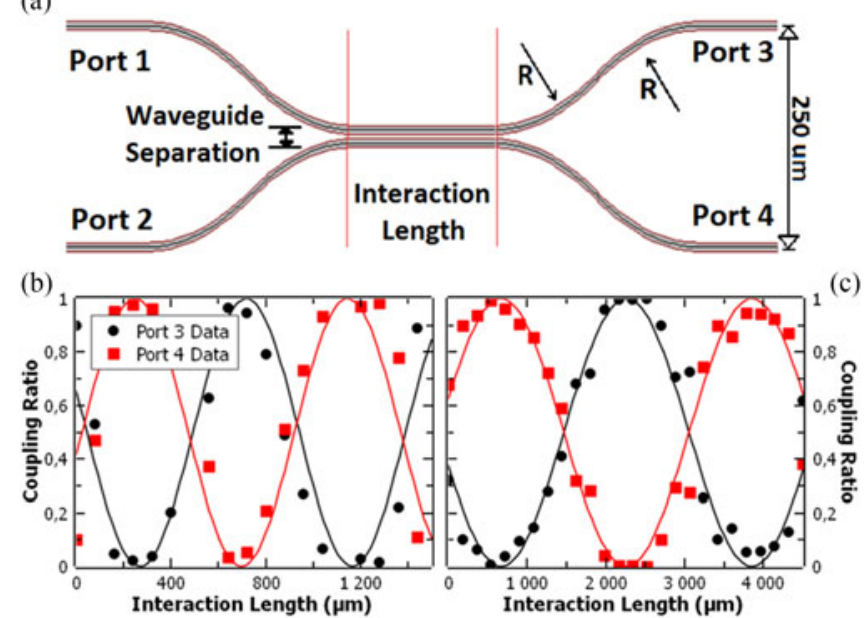

(d)

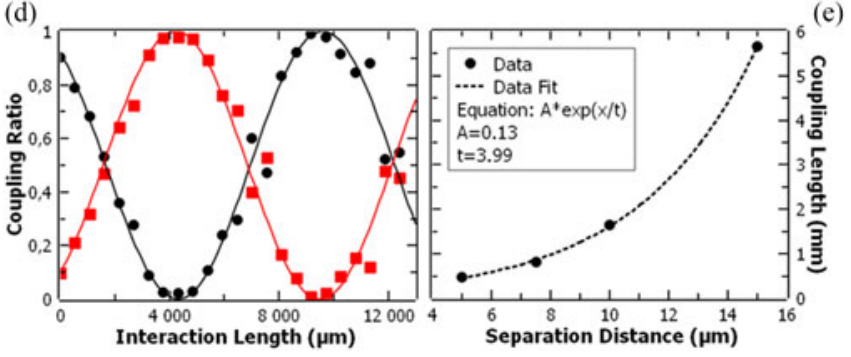

Fig. 6. (a) Directional couplers design. Coupling ratio for directional couplers with separation distances of (b) $5 \mu \mathrm{m}$, (c) $10 \mu \mathrm{m}$, and (d) $15 \mu \mathrm{m}$ as a function of interaction length, for port 1 as input. The solid lines represent the fit of the theoretical coupling ratio equation to the experimental data. (e) Coupling length and respective exponential growth fit as a function of the tested separation distance.

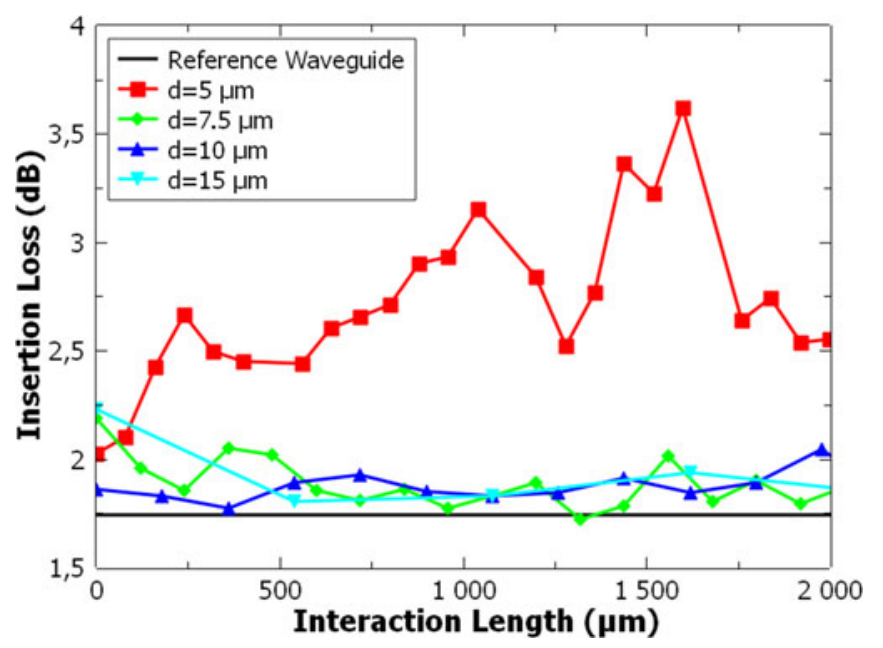

Fig. 7. Insertion loss for directional couplers with a separation distance of $5,7.5,10$ and $15 \mu \mathrm{m}$ as a function of interaction length. Insertion loss of a reference waveguide is also displayed.

separation distance of $5 \mu \mathrm{m}$ the average insertion loss increases with interaction length, which indicates a degradation of the interacting waveguides probably due to an overlapping of the material modification. When the separation distance increases such overlapping ceases to exist and guiding occurs without any major additional loss from the single waveguide case, as seen

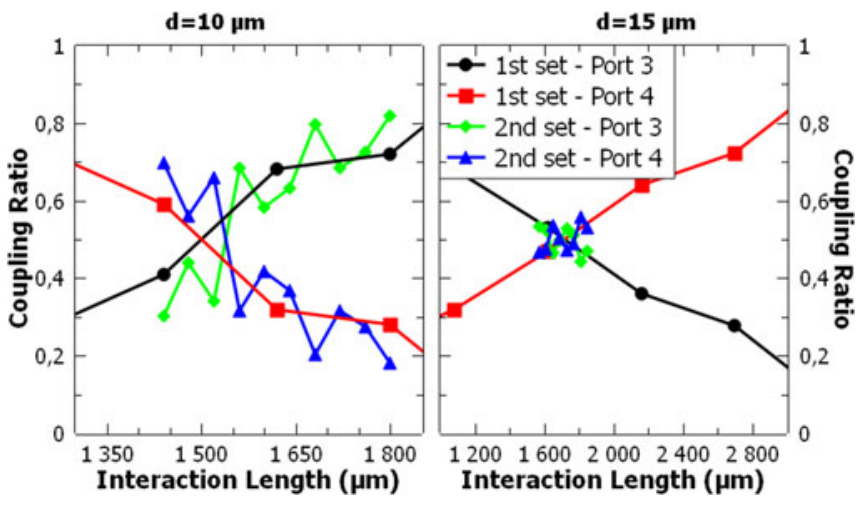

Fig. 8. Coupling ratio as a function of interaction length for directional couplers fabricated with a separation distance of 10 and $15 \mu \mathrm{m}$. The first set corresponds to the data displayed in Fig. 6 and the second set corresponds to the devices fabricated to more accurately find the $3 \mathrm{~dB}$ location.

for separation distances of 7.5, 10 and $15 \mu \mathrm{m}$. The insertion loss fluctuation for each of the fabricated devices (in particular the high loss registered for a separation of 7.5 and $15 \mu \mathrm{m}$ at a zero interaction length) may be associated with the quality of the polishing along the facets, non-homogeneities in the substrate material and defects at the time of fabrication, due to external vibrations, for instance.

Finally, the repeatability of directional couplers was tested for separation distances of 10 and $15 \mu \mathrm{m}$ while searching the interaction length that results in $3 \mathrm{~dB}$ splitting. Fig. 8 shows the coupling ratio results of directional couplers depicted in Fig. 6 as well as a new set of directional couplers fabricated in $40 \mu \mathrm{m}$ interaction length intervals around the length necessary for $3 \mathrm{~dB}$ splitting. A higher variation was found for a smaller separation distance, as expected, since any fluctuation in separation distance while fabricating the devices causes a performance deviation that is larger for higher coupling coefficients.

From this study it was possible to obtain directional couplers with a splitting ratio of $44 / 56 \pm 1 \%$, for a separation distance of $10 \mu \mathrm{m}$ and an interaction length of $1.48 \mathrm{~mm}$, and 51/49 $\pm 1 \%$, for a separation distance of $15 \mu \mathrm{m}$ and an interaction length of $1.77 \mathrm{~mm}$. For the fabrication of OADMs a separation distance of $15 \mu \mathrm{m}$ was implemented since, as already seen, the coupling ratio fluctuations are strongly decreased, enabling the fabrication of reproducible directional couplers with a high splitting uniformity.

\section{Add-Drop Multiplexers}

To fabricate a complete OADM on a single chip, the previously discussed optical components (waveguides, BGWs, and directional couplers) were integrated in the MZI configuration represented in Fig. 1. The device was fabricated in a $75 \mathrm{~mm}$ long pure silica substrate and the fabrication process took around 13 minutes to finish. Straight sections were added in both ends so the device uses the total substrate length. The substrate containing the final device was cut to a length of $60 \mathrm{~mm}$ and the end facets polished. After the polishing, the complete device was $54 \mathrm{~mm}$ long ( $28 \mathrm{~mm}$ for the directional couplers, 20 $\mathrm{mm}$ for the BGWs and the remaining for straight waveguides 


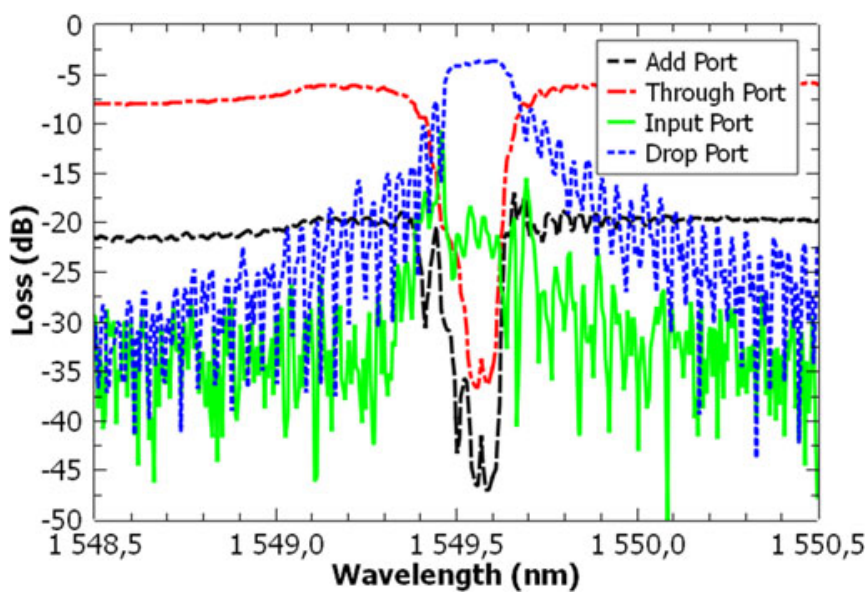

Fig. 9. Measured reflection and transmission spectra of the input, drop, add, and through-port of the fabricated OADM.

connecting the components above). V-grooves were glued to the final device with NOA 61 using an UV light source.

The results presented here show a device that had a good phase balance as written; real time phase trimming was not tested yet. The input, drop, add, and through-port spectra around the Bragg wavelength are shown in Fig. 9, with a resolution of $5 \mathrm{pm}$, for a TE polarization (horizontally polarized light). The spectra for TM polarization are not shown since the behavior is the same as for TE polarization, with a shift in wavelength due to the gratings' birefringence and an increased insertion loss due to polarization dependent loss. As can be seen, the reflected signal is emerging from the input and drop-ports with an extinction ratio of approximately $14 \mathrm{~dB}$ between ports, showing that the phase difference between the two arms of the interferometer is around 11.5 degrees (plus multiples of 360 degrees). A similar behavior occurs for the transmitted signal.

Regarding the transmission spectra, at the Bragg wavelength an isolation better than $-30 \mathrm{~dB}$ is achieved, enabling low intrachannel crosstalk. On the other hand, the reflection spectrum at the drop-port corresponds to the usual uniform Bragg grating spectrum with the undesired side lobes. The $3 \mathrm{~dB}$ bandwidth is $0.19 \pm 0.01 \mathrm{~nm}$ and a side lobe suppression of $20 \mathrm{~dB}$ is achieved for $\Delta \lambda= \pm 0.75 \mathrm{~nm}$.

Also in the transmission spectra, the short wavelength side clearly shows loss due to the coupling into radiation modes by the BGWs, similar to the loss observed in the coupling into cladding modes in fiber Bragg gratings. The size of this unwanted loss increases with grating strength and reaches $\sim 2 \mathrm{~dB}$ for a grating strength of $30 \mathrm{~dB}$. These out-of-band losses affect the usability and performance of such devices since channels with a lower wavelength will suffer unintentional extra loss. To avoid this characteristic the channels at lower wavelengths should be dropped first, meaning that the power of the next, higher wavelength, channels is unaltered. However, this solution has obvious limitations. Improvements can be obtained by optimizing the BGWs to minimize the coupling to radiation modes. This can be achieved by increasing the duty cycle of the writing beam, which leads to a decrease in the coupling coefficient but also in a decrease in the out-of-band loss. The
TABLE I

ADD-DROP MULTIPLEXER COMPARISON

\begin{tabular}{|c|c|c|c|c|}
\hline Symbol & $\begin{array}{l}\text { Kashyap } \\
\text { et al. [20] }\end{array}$ & $\begin{array}{l}\text { Albert et } \\
\text { al. }[21]\end{array}$ & $\begin{array}{l}\text { Kim et } \\
\text { al. [22] }\end{array}$ & $\begin{array}{l}\text { This } \\
\text { paper }\end{array}$ \\
\hline $\begin{array}{l}\text { Intra-channel } \\
\text { Crosstalk }(\mathrm{dB})\end{array}$ & -15 & -36 & -24 & -30 \\
\hline 3 dB Bandwidth (nm) & 1 & 1.1 & 0.5 & 0.19 \\
\hline Grating Length (mm) & 2 & 3.8 & 10 & 20 \\
\hline $\begin{array}{c}\text { Input/Drop } \\
\text { Extinction Ratio (dB) }\end{array}$ & 7.8 & 10 & $\mathrm{n} / \mathrm{a}$ & 14 \\
\hline $\begin{array}{c}\text { Add/Through } \\
\text { Extinction Ratio }(\mathrm{dB})\end{array}$ & 14.3 & 10 & 20.5 & 14 \\
\hline Device Length (cm) & $\mathrm{n} / \mathrm{a}$ & 2.5 & 3 & 5.4 \\
\hline $\begin{array}{c}\text { Through Port } \\
\text { Insertion Loss }(\mathrm{dB})\end{array}$ & 1.5 & 1 & $2.5^{*}$ & 4.9 \\
\hline $\begin{array}{c}\text { Drop Port } \\
\text { Insertion Loss }(\mathrm{dB})\end{array}$ & 2.3 & 1 & $\mathrm{n} / \mathrm{a}$ & 3.7 \\
\hline
\end{tabular}

*without taking into account coupling loss to an optical fiber

disadvantage of using this approach is the need for a longer $\mathrm{BGW}$, which will increase the overall size of the device.

A total insertion loss of $4.9 \mathrm{~dB}$ and $3.7 \mathrm{~dB}$ was measured for the through port and the drop port respectively. This result was unexpected since by taking into account the individual losses of all components a total through-port insertion loss of $3 \mathrm{~dB}$ was expected. The measured insertion losses could be explained by the leakage of optical power to the add and input ports, due to the MZI unbalance, together with an unanticipated deviation from the optimal fabrication parameters of the waveguides and/or BGWs, as well as a small misalignment of the glued v-grooves caused by shrinkage of the glue itself during the curing process.

A comparison of this device performance with other MZI OADMs reported on the literature is given on Table I. In [20] and [21], two MZI OADMs are fabricated with standard lithographic techniques together with UV writing, while in [22] an ion exchange with an overlay grating is used. The device reported here exhibited a lower than most intra-channel crosstalk of $-30 \mathrm{~dB}$, with a much lower $3 \mathrm{~dB}$ bandwidth of $0.19 \pm$ $0.01 \mathrm{~nm}$, a similar extinction ratio of $14 \mathrm{~dB}$ without phase trimming, and a higher insertion loss of $3.7 \mathrm{~dB}$ and $4.9 \mathrm{~dB}$ for the drop and through port respectively (using TE polarized light), as a result of a $5.4 \mathrm{~cm}$ long device.

Future improvement of this device is related to the fine tuning of the ports output by compensating phase errors with resource to real time laser trimming. The same laser used to write the waveguides should be used to adjust the optical paths, first optimizing the drop port and after the through port. This process might be accomplished with the modification of the already manufactured arms of the MZI [20] or by the fabrication of stress tracks parallel to one of the MZI arms [17]. Also, the grating profile should be optimized in order to enhance, for example, channel crosstalk. This can be done through apodization [18].

\section{CONCLUSION}

The fabrication of OADM devices, working on the $\mathrm{C}$ band, is reported for the first time to our knowledge with the femtosecond laser direct writing technique. The device was built 
using a MZI configuration, and as such the fabrication of $3 \mathrm{~dB}$ couplers and uniform first order Bragg gratings was optimized. An optimum splitting ratio of $51 / 49 \pm 1 \%$ was obtained on the directional couplers for a separation distance of $15 \mu \mathrm{m}$ and an interaction length of $1.77 \mathrm{~mm}$, enabling optimal routing of the signal throughout the device. Regarding the first order Bragg grating waveguides, an AOM duty cycle of $40 \%$ was used in the fabrication as a compromise between grating strength and propagation loss. Although phase errors still remain an issue, the fabricated OADM showed a $3 \mathrm{~dB}$ bandwidth of $0.19 \pm$ $0.01 \mathrm{~nm}$, with an intra-channel and adjacent inter-channel crosstalk of -30 and $-20 \mathrm{~dB}$, respectively.

\section{REFERENCES}

[1] J. Wang and L. R. Chen, "Low crosstalk Bragg grating/Mach-Zehnder interferometer optical add-drop multiplexer in silicon photonics," Opt. Express, vol. 23, no. 20, pp. 26450-26459, Oct. 2015.

[2] G. Della Valle, R. Osellame, and P. Laporta, "Micromachining of photonic devices by femtosecond laser pulses," J. Opt. A, Pure Appl. Opt., vol. 11, no. 1, Dec. 2008, Art. no. 013001.

[3] S. M. Eaton, H. Zhang, and P. R. Herman, "Heat accumulation effects in femtosecond laser-written waveguides with variable repetition rate," Opt. Express, vol. 13, no. 12, pp. 4708-4716, Jun. 2005.

[4] H. Zhang, S. M. Eaton, and P. R. Herman, "Single-step writing of Bragg grating waveguides in fused silica with an externally modulated femtosecond fiber laser," Opt. Lett., vol. 32, no. 17, pp. 2559-2561, Sep. 2007.

[5] S. M. Eaton et al., "Telecom-band directional coupler written with femtosecond fiber laser," IEEE Photon. Technol. Lett., vol. 18, no. 20, pp. 2174-2176, Oct. 2006.

[6] V. A. Amorim, J. M. Maia, D. Alexandre, and P. V. S. Marques, "Optimization of broadband Y-junction splitters in fused silica by femtosecond laser writing," IEEE Photon. Technol. Lett., vol. 29, no. 7, pp. 619-622, Apr. 2017.

[7] T. Calmano, J. Siebenmorgen, O. Hellmig, K. Petermann, and G. Huber, "Nd:YAG waveguide laser with $1.3 \mathrm{~W}$ output power, fabricated by direct femtosecond laser writing," Appl. Phys. B, vol. 100, no. 1, pp. 131-135, Jul. 2010.

[8] R. R. Thomson et al., "Ultrafast-laser inscription of a three dimensional fan-out device for multicore fiber coupling applications," Opt. Express, vol. 15 , no. 18 , pp. 11691-11697, Sep. 2007.

[9] B. Guan et al., "Free-space coherent optical communication with orbital angular, momentum multiplexing/demultiplexing using a hybrid 3D photonic integrated circuit," Opt. Express, vol. 22, no. 1, pp. 145-156, Jan. 2014

[10] A. Crespi et al., "Three-dimensional Mach-Zehnder interferometer in a microfluidic chip for spatially-resolved label free detection," Lab Chip, vol. 10, no. 9, pp. 1167-1173, May 2010.

[11] N. Bellini et al., "Femtosecond laser fabricated monolithic chip for optical trapping and stretching of single cells," Opt. Express, vol. 18, no. 5, pp. 4679-4688, Mar. 2010.

[12] R. Osellame, G. Cerullo, and R. Ramponi, "Controlling the cross-section of ultrafast laser inscribed optical waveguides," in Femtosecond Laser Micromachining: Photonic and Microfluidic Devices in Transparent Materials, 1st ed. New York, NY, USA: Springer, 2012, ch. 5, pp. 93-122.

[13] Y. Nasu, M. Kohtoku, and Y. Hibino, "Low-loss waveguides written with a femtosecond laser for flexible interconnection in a planar light-wave circuit," Opt. Lett., vol. 30, no. 7, pp. 723-725, Apr. 2005.

[14] T. Fukuda, S. Ishikawa, T. Fujii, K. Sakuma, and H. Hosoya, "Lowloss optical waveguides written by femtosecond laser pulses for threedimensional photonic devices," Proc. SPIE, vol. 5339, pp. 524-538, Jul. 2004.

[15] S. M. Eaton, M. L. Ng, R. Osellame, and P. R. Herman, "High refractive index contrast in fused silica waveguides by tightly focused, high-repetition rate femtosecond laser," J. Non-Cryst. Solids, vol. 357, no. 11-13, pp. 2387-2391, Jun. 2011.

[16] R. Taylor, C. Hnatovsky, and E. Simova, "Applications of femtosecond laser induced self-organized planar nanocracks inside fused silica glass," Laser Photon. Rev., vol. 2, no. 1/2, pp. 26-46, Jan. 2008.

[17] L. A. Fernandes, J. R. Grenier, P. R. Herman, J. S. Aitchison, and P. V. S. Marques, "Stress induced birefringence tuning in femtosecond laser fabricated waveguides in fused silica," Opt. Express, vol. 20, no. 22 , pp. 24103-24114, Oct. 2012.

[18] P. Zeil, C. Voigtländer, J. Thomas, D. Richter, and S. Nolte, "Femtosecond laser-induced apodized Bragg grating waveguides," Opt. Lett., vol. 38, no. 13, pp. 2354-2356, Jul. 2013.

[19] M. Ams, P. Dekker, S. Gross, and M. J. Withford, "Fabricating waveguide Bragg gratings (WBGs) in bulk materials using ultrashort laser pulses," Nanophotonics, to be published.

[20] R. Kashyap, G. D. Maxwell, and B. J. Ainslie, "Laser-trimmed four-port bandpass filter fabricated in single-mode photosensitive Ge-doped planar waveguide," IEEE Photon. Technol. Lett., vol. 5, no. 2, pp. 191-194, Feb. 1993.

[21] J. Albert et al., "Low-loss planar lightwave circuit OADM with high isolation and no polarization dependence," IEEE Photon. Technol. Lett. vol. 11, no. 3, pp. 346-348, Mar. 1999.

[22] J. Kim, G. Li, and K. A. Winick, "Design and fabrication of a glass waveguide optical add-drop multiplexer by use of an amorphous-silicon overlay distributed Bragg reflector," Appl. Opt., vol. 43, no. 3, pp. 671677, Jan. 2004

Vítor A. Amorim was born in Barcelos, Portugal, in 1993. He received the B.Sc degree in technological physics and the M.Sc. degree in engineering physics from the University of Porto, Portugal in 2014 and 2016 respectively, on the fabrication of integrated optical devices in fused silica using the femtosecond laser direct writing technique. Since 2016, he has been working toward the Ph.D degree in physics from the University of Porto. In 2015, he joined the Centre for Applied Photonics, Instituto de Engenharia de Sistemas e ComputadoresTecnologia e Ciência, Porto, Portugal, where he is currently enrolled in research activity. His research interests include integrated optical devices and monolithic optofluidic devices fabricated by femtosecond laser direct writing techniques for bio- and chemical sensing.

João M. Maia was born in Porto, Portugal, in 1993. He received the B.Sc. degree in technological physics and the M.Sc. degree in physics engineering, both from the University of Porto, Porto, Portugal, in 2014 and 2016, respectively. Since September 2016, he has been working toward the Ph.D. degree in physics from the University of Porto.

In 2015, he joined the Centre for Applied Photonics , Instituto de Engenharia de Sistemas e Computadores-Tecnologia e Ciência, Porto, Portugal, where he is currently enrolled in research activity. His research interest focuses on fabrication of optofluidic devices by femtosecond laser micromachining for chemical and biosensing.

Daniel Alexandre was born in Viseu, Portugal, in 1976. He received the degree in electrotechnics engineering from the University of Trás-os-Montes e Alto Douro, Vila Real, Portugal, in 1999, and the M.Sc. degree in optoelectronics and lasers and the Ph.D. degree in physics, both from the University of Porto, Porto, Portugal, in 2003 and 2011, respectively. His Ph.D. research programme was conducted under a strong collaboration with the Centre for Applied Photonics (CAP) at, Instituto de Engenharia de Sistemas e Computadores-Tecnologia e Ciência (INESC TEC), Porto, Portugal, and it involved the development and characterization of passive and active integrated optical devices using hybrid sol-gel and laser direct writing.

In March 1999, he started teaching as a Monitor in the Physics Department, University of Trás-os-Montes e Alto Douro, where he was appointed an Assistant Professor in 2011. Since 2002, he has been developing research activity in the CAP, INESC TEC. His research interests include integrated optical devices and microfluidics, Bragg gratings, integrated optical sensors, and laser direct writing techniques for integrated optics and microfabrication

Paulo V. S. Marques is an Assistant Professor in the Physics and Astronomy Department, Faculty of Science of Porto University, Porto, Portugal. Since October 2009, he is the Coordinator of the Center of Applied Photonics, Instituto de Engenharia de Sistemas e Computadores-Tecnologia e Ciência, Porto, Portugal, and since May 2013, he has been the Director of the Micro- and Nanofabrication Center, Porto University (CEMUP MNTEC).

He has published four world patents (patent family of 32), more than 100 scientific papers in international magazines, and conferences and three book chapters. His research interests include optical sensors, femtosecond laser direct writing techniques for integrated optics and microfabrication in general microfluidics, Bragg gratings, and active devices. 